\title{
Fulvestrant as a reference antiestrogen and estrogen receptor (ER) degrader in preclinical studies: treatment dosage, efficacy, and implications on development of new ER-targeting agents
}

\author{
Guangdi Wang \\ RCMI Cancer Research Center, Xavier University of Louisiana, New Orleans, LA, USA \\ Correspondence to: Guangdi Wang. RCMI Cancer Research Center, Xavier University of Louisiana, New Orleans, LA 70125, USA. \\ Email: guangdi.wang@zenopharm.com. \\ Provenance and Peer Review: This article was commissioned by the editorial office, Translational Cancer Research. The article did not undergo external \\ peer review. \\ Comment on: Wardell SE, Yllanes AP, Chao CA, et al. Pharmacokinetic and pharmacodynamic analysis of fulvestrant in preclinical models of breast \\ cancer to assess the importance of its estrogen receptor- $\alpha$ degrader activity in antitumor efficacy. Breast Cancer Res Treat 2020;179:67-77.
}

Submitted Jun 01, 2020. Accepted for publication Jun 30, 2020.

doi: $10.21037 /$ tcr-20-2166

View this article at: http://dx.doi.org/10.21037/tcr-20-2166

Endocrine therapy remains the standard of care for hormone receptor positive (HR+), HER2 negative (HER2-) breast cancer. Clinically approved endocrine agents include selective estrogen receptor modulators (SERMs), aromatase inhibitors (AIs), and selective estrogen receptor downregulators/degraders (SERDs). These therapeutic regimens are well tolerated with manageable side effects and have proven effective in early stage breast cancer with curative intent. In the metastatic or advanced setting, endocrine therapy improves patient survival and quality of life, with eventual resistance development and disease progression. Fulvestrant is the only FDA approved SERD indicated for metastatic/advanced $\mathrm{HR}+$ breast cancer. In 2002, fulvestrant was first approved as a second line endocrine treatment for patients with disease progression on first line antiestrogen therapy following two phase III studies comparing the efficacy and safety of fulvestrant with anastrozole (1). In 2017, 15 years after the first approval, fulvestrant was approved as a first line agent for patients with advanced or metastatic breast cancer $(2,3)$. This approval came after a pivotal clinical trial (FALCON) comparing the efficacy of anastrozole and $500 \mathrm{mg}$ fulvestrant in endocrine naïve patients, which demonstrated that fulvestrant treated patients had a significantly longer progression free survival (PFS) and overall survival (OS) than those taking anastrozole (4). Fulvestrant is also the first endocrine agent to be approved in combination therapy with CDK4/6 inhibitors for metastatic/advanced breast cancer (5).

Fulvestrant was developed as a pure antiestrogen by modification of long-chain alkyl substitutes in the $7 \alpha$-position of estradiol $(6,7)$. The steroidal antiestrogen was shown to have no estrogen receptor agonist or partial agonist effects in any species or organ where other antiestrogens show tissue-selective agonistic activities such as tamoxifen and raloxifene. Fulvestrant is able to overcome the estrogenic effects of estradiol and tamoxifen in preclinical models in a dose-dependent manner. In xenograft tumor models, fulvestrant also demonstrates superior antitumor activity compared with tamoxifen. The ability of fulvestrant to reduce the cellular content of the estrogen receptor by reducing the half-life of the protein was confirmed in clinical trials $(8,9)$. Importantly, the decrease in ER protein level by treatment of fulvestrant did not involve a change at the mRNA level (9).

The clinical efficacy of fulvestrant and the apparent lack of cross-resistance to tamoxifen and AIs are attributed to its distinct mode of action, namely the pure antiestrogenic properties of the drug. This mode of action is shown to be the result of a unique, unstable ER conformation upon binding with fulvestrant, which inhibits ER dimerization and nuclear translocation $(10,11)$. But because this conformational change rapidly leads to proteosomal ER degradation, fulvestrant's deeper and more durable blockade 
of ER-dependent signaling in breast cancer is therefore considered a result of the ultimate downregulation of the nuclear receptor at the protein level (12).

Despite the establishment of fulvestrant as an effective second line endocrine treatment in 2002, and recently as a first line therapy superior to anastrozole for advanced HR+ breast cancer $(2,3)$, its poor pharmacokinetic properties and insufficient drug exposure are believed to limit clinical response that could be further improved. Indeed, efforts to increase fulvestrant exposure through multiple clinical trials between 2005 and 2009 led to subsequent approval of a higher dosage fulvestrant in 2010 (13). Still, clinical studies indicate that at $500 \mathrm{mg}$, the maximum injectionable limit has been reached, but the maximum therapeutic effect has not (14). Thus, a potent, orally bioavailable SERD that could achieve higher steady-state drug levels more rapidly would have the potential for increased receptor knockdown and lead to quicker clinical responses, reducing the possibility of early relapses. This unmet clinical need has driven extensive efforts to develop oral SERDs in the past ten years, culminating in as many as 13 oral SERDs entering clinical trials (NCT01823835, NCT02316509, NCT02248090, NCT03916744, NCT04214288, NCT02734615, NCT03284957, NCT03455270, NCT04188548, NCT03471663, NCT03596658, NCT04176757, NCT02338349). From available study reports describing the discovery and development of these oral SERDs, which are invariably non-steroidal small molecules characterized by an ER binding motif and a side chain featuring either an acrylic acid or an amino base terminal that confer antiestrogenic and ER degrading activities. As oral SERDs were sought to improve upon fulvestrant, a full antagonist and ER downregulator, optimization of ER degradation has been the strategy to identify lead compounds that possess fulvestrant-like mode of action, and preclinical studies of oral SERDs typically employ fulvestrant as a standard of reference in the evaluation of efficacy and bioavailability. While in vitro pharmacology studies compare dose-dependent activities of candidate oral SERDs with those of fulvestrant, in vivo efficacy studies often measure the efficacy of oral SERDs at various doses in comparison to that of fulvestrant treatment group given a widely adopted dose of $200 \mathrm{mg} / \mathrm{kg}$ via s.c. injection.

The study by Wardell et al. (15) asked the question whether such dose of fulvestrant is appropriate in the development stage of oral SERDs involving parallel efficacy comparisons. The study investigated drug exposure and intratumoral ER downregulation at varying doses of fulvestrant administered to nude mice bearing three xenograft models of endocrine therapy-resistant breast cancer to evaluate the efficacy of fulvestrant and GDC0810, AZD9496, and bazedoxifene. The study found that antitumor efficacy could be achieved with a clinically relevant $25 \mathrm{mg} / \mathrm{kg}$ weekly injection dose of fulvestrant that is comparable to the widely used $200 \mathrm{mg} / \mathrm{kg}$ dose. At the clinically relevant dose fulvestrant enabled modest ER downregulation in tumor tissue, roughly $50 \%$ in the $\mathrm{H} 1428$ and LTED tumor model, and 30-40\% downregulation in the TamR model. When compared with GDC-0810 and AZD9496, the antitumor efficacy of the lower dose of fulvestrant was similar to the oral SERDs. The study suggests that using clinically unachievable exposure levels of fulvestrant as a benchmark in preclinical development of SERDs may negatively impact the selection of clinical candidates. Further, the study suggests that the antiestrogenic action of fulvestrant is the main drive for its anticancer efficacy, based on the observation that efficacy was achieved when ER downregulation was incomplete and proposes that antiestrogenic efficacy conferred by drug exposure and antagonistic target engagement, rather than capacity of ER downregulation should be considered in development of oral SERDs.

SERD-like properties were first reported in a nonsteroidal, tamoxifen-like molecule known as GW5638 (16), although the compound was initially developed to spare uterotrophic effect while acting as an antagonist in mammary cells. Importantly, GW5638 was found to be effective in tamoxifen resistant breast cancer, had no agonist effect in the uterus, and degraded ER by conformational changes that increased hydrophobic surface and instability of ER. A study by Fan et al. showed that the acrylic acid moiety in GW5638 was key to impart the ER downregulation activity (17). These findings informed further development of structurally similar, preclinically improved oral SERDs that entered clinical trials beginning in 2013 (NCT01823835, NCT02248090), more than a decade after the inconclusive clinical trial of GW5638 (18). The oral SERDs with disclosed structures are shown in Figure 1, all of which are nonsteroidal.

The preclinical development of GDC-0810 was well documented $(19,20)$ where the drug candidate was evaluated extensively in mode of action validation, pharmacokinetics, pharmacodynamics, and efficacy in endocrine resistant breast cancer models. Notably, GDC-0810 was tested against fulvestrant, GW5638, and SERMS for effects on 


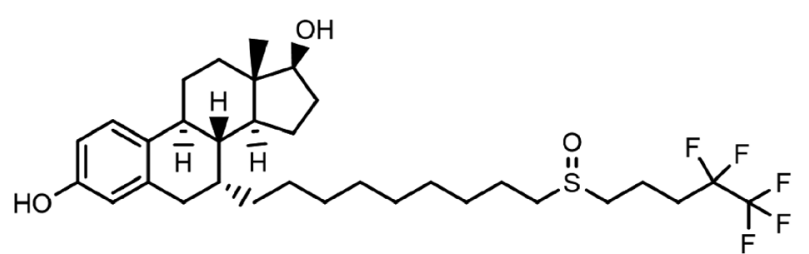

Fulvestrant<smiles>CCC(=C(c1ccccc1)c1ccccc1)c1ccc(/C=C/C(=O)O)cc1</smiles>

GW5638<smiles>CC/C(=C(\c1ccc(/C=C/C(=O)O)cc1)c1ccc2[nH]ncc2c1)c1ccc(F)cc1Cl</smiles>

GDC-810<smiles>C[C@H]1Cc2c([nH]c3ccccc23)[C@@H](c2c(F)cc(/C=C/C(=O)O)cc2F)N1CC(C)(C)F</smiles>

AZD9496<smiles>Cc1cc(F)cc(C)c1C(=O)c1sc2cc(O)ccc2c1Oc1ccc(/C=C/C(=O)O)cc1</smiles>

G1T48<smiles>CC(F)(F)c1cc(F)ccc1-c1sc2cc(O)ccc2c1Oc1ccc(/C=C/C(=O)O)cc1</smiles>

LSZ102<smiles>CC1=C(c2cccc(O)c2)[C@@H](c2ccc(OCCN3CC(CF)C3)cc2)Oc2ccc(O)cc21</smiles>

GDC-0927

\begin{tabular}{lll}
\hline Oral SERD & Developmental Stage & Reference \\
\hline GW5638 & Phase 1 (2001-2002) & J Med Chem 2015; 58:4883-7 \\
\hline GDC-0810 & $\begin{array}{l}\text { Phase 1 (NCT01823835), phase 2 } \\
\text { (NCT02569801, terminated) }\end{array}$ & $\begin{array}{l}\text { J Med Chem 2015; 58:4888-904 } \\
\text { Clin Cancer Res. 2017; 23:3053-3060 }\end{array}$ \\
\hline GDC-0927 & Phase 1 (NCT02316509) & $\begin{array}{l}\text { Cell 2019; 178:949-963 } \\
\text { ACS Med Chem Lett 2018;10:50-55 }\end{array}$ \\
& & J Med Chem 2015; 58:8128-40 \\
\hline AZD9496 & Phase 1 (NCT02248090, NCT03236974) & Breast Cancer Res Treat 2020;180:635-6 \\
\hline G1T48 & Phase 1 (NCT03455270) & J Med Chem 2018; 6:2837-2864 \\
\hline LSZ102 & Phase 1/1b (NCT02734615) & J Med Chem 2020; 63:512-528 \\
SAR439859 & Phase 1/2 (NCT03284957), Phase 2 & \\
\hline RAD1901 & Phase 3 (NCT03778931) & $\begin{array}{l}\text { Endocr Relat Cancer 2015;22:713-24 } \\
\text { Clin Cancer Res 2017;23:4793-4804 }\end{array}$ \\
\hline
\end{tabular}

Figure 1 Structures of fulvestrant and oral SERDs under clinical development. SERDs, selective estrogen receptor downregulators/ degraders. 
transcriptional activity, ER binding, ER degradation, tissue selectivity, and xenograft tumor growth $(19,20)$. Fulvestrant dose ranged from 25 to $200 \mathrm{mg} / \mathrm{kg}$ in diverse clinically relevant tumor models in which GDC-0810 efficacy was found to be comparable to or better than that achieved by the highest fulvestrant dose. The use of high dose fulvestrant as a comparison group in the preclinical evaluation of GDC-0810 unlikely served as a benchmark criterion over which a drug candidate made the cut. Rather, the maximum achievable efficacy in animal models by fulvestrant provided a measure for the drug exposure needed for the oral SERD candidate to achieve similar or better results. Even so, when GDC-0810 was compared with fulvestrant in a phase 2 trial (NCT02569801), it failed to show comparable or superior efficacy and the study was terminated.

Similarly, in the preclinical development of AZD9496, fulvestrant was employed as a benchmark reference in all in vitro and in vivo pharmacological studies of the oral SERD candidate $(21,22)$. AZD9496 was shown to bind both wild type and mutant ER with greater affinity than fulvestrant, inhibit ER+ breast cancer cell proliferation more potently than fulvestrant, and blocking tumor growth in endocrine resistant, ESR1 mutant breast cancer models more efficaciously than fulvestrant. In a diverse panel of clinically relevant breast tumor models, AZD9496 consistently demonstrated superior efficacy when compared with fulvestrant administered at $200 \mathrm{mg} / \mathrm{kg}$, a clinically unachievable dose and drug exposure. Despite this preclinically observed advantage over fulvestrant, in a randomized, window of opportunity study comparing AZD9496 with fulvestrant in patients with ER+, HER2primary breast cancer, the oral SERD was inferior to fulvestrant in both anticancer efficacy and reduction of ER and PR expression (23).

As oral SERDs, all of the investigational new drug molecules that have been developed so far possess the dual mechanisms of action: selective ER antagonism and ER degradation. Indeed, a pure antiestrogen that does not significantly downregulate the estrogen receptor has yet to be identified. Conversely, a pure ER degrading agent, in the form of PROTAC, has been developed into phase 1 clinical trial (NCT04072952). In this case, the ER-binding warhead can be a SERM moiety which does not induce hydrophobic surface exposure and result in receptor degradation. Rather, the other end of the PROTAC molecule engages an E3 ligase to ubiquitinate $\mathrm{ER}$ and degrades it in a catalytic manner. Thus pure antagonism of ER is realized by elimination of the receptor, rather than conformational changes of ER to block recruitment of co-factors required for ER transcriptional activation.

\section{Acknowledgments}

Funding: NIH National Institute on Minority Health and Health Disparities (NIMHD) Grant U54MD007595.

\section{Footnote}

Conflicts of Interest: The author has completed the ICMJE uniform disclosure form (available at http://dx.doi. org/10.21037/tcr-20-2166). GW reports grants from Zenopharm, LLC, during the conduct of the study; grants from Zenopharm, LLC, outside the submitted work; in addition, Dr. GW has a patent A boron-based prodrug strategy for increased bioavailability and lowerdosage requirements for drug molecules containing at least one phenol (or aromatic hydroxyl) group licensed to Zenopharm, LLC, and a patent selective estrogen receptor downregulators (SERDS) licensed to Zenopharm, LLC.

Ethical Statement: The author is accountable for all aspects of the work in ensuring that questions related to the accuracy or integrity of any part of the work are appropriately investigated and resolved.

Open Access Statement: This is an Open Access article distributed in accordance with the Creative Commons Attribution-NonCommercial-NoDerivs 4.0 International License (CC BY-NC-ND 4.0), which permits the noncommercial replication and distribution of the article with the strict proviso that no changes or edits are made and the original work is properly cited (including links to both the formal publication through the relevant DOI and the license). See: https://creativecommons.org/licenses/by-nc-nd/4.0/.

\section{References}

1. Bross PF, Cohen MH, Williams GA, et al. FDA drug approval summaries: fulvestrant. Oncologist 2002;7:477-80.

2. Available online: https://www.astrazeneca.com/mediacentre/press-releases/2017/faslodex-receives-euapproval-as-first-line-therapy-for-advanced-breastcancer-26072017.html

3. Available online: https://www.astrazeneca.com/mediacentre/press-releases/2017/faslodex-receives-us-fdaapproval-as-monotherapy-for-expanded-use-in-breast- 
cancer.html

4. Robertson JFR, Bondarenko IM, Trishkina E, et al. Fulvestrant $500 \mathrm{mg}$ versus anastrozole $1 \mathrm{mg}$ for hormone receptor-positive advanced breast cancer (FALCON): an international, randomised, double-blind, phase 3 trial. Lancet 2016;388:2997-3005.

5. Available online: https://www.fda.gov/Drugs/ InformationOnDrugs/ApprovedDrugs/ucm487080.htm

6. Wakeling AE, Bowler J. Steroidal pure antioestrogens. J Endocrinol 1987;112:R7-10.

7. Wakeling AE, Bowler J. Biology and mode of action of pure antioestrogens. J Steroid Biochem 1988;30:141-7.

8. DeFriend DJ, Howell A, Nicholson RI, et al. Investigation of a new pure antiestrogen (ICI 182780) in women with primary breast cancer. Cancer Res 1994;54:408-14.

9. McClelland RA, Manning DL, Gee JM, et al. Effects of short-term antiestrogen treatment of primary breast cancer on estrogen receptor mRNA and protein expression and on estrogen-regulated genes. Breast Cancer Res Treat 1996;41:31-41.

10. Parker MG. Action of "pure" antiestrogens in inhibiting estrogen receptor action. Breast Cancer Res Treat 1993;26:131-7.

11. Dauvois S, White R, Parker MG. The antiestrogen ICI 182780 disrupts estrogen receptor nucleocytoplasmic shuttling. J Cell Sci 1993;106:1377-88.

12. Wittmann BM, Sherk A, McDonnell DP. Definition of functionally important mechanistic differences among selective estrogen receptor down-regulators. Cancer Res 2007;67:9549-60.

13. Di Leo A, Jerusalem G, Petruzelka L, et al. Final overall survival: fulvestrant $500 \mathrm{mg}$ vs $250 \mathrm{mg}$ in the randomized CONFIRM trial. J Natl Cancer Inst 2014;106:djt337.

14. Robertson JF, Lindemann J, Garnett S, et al. A good drug made better: the fulvestrant dose-response story. Clin Breast Cancer 2014;14:381-9.

15. Wardell SE, Yllanes AP, Chao CA, et al. Pharmacokinetic and pharmacodynamic analysis of fulvestrant in preclinical models of breast cancer to assess the importance of its estrogen receptor- $\alpha$ degrader activity in antitumor efficacy. Breast Cancer Res Treat 2020;179:67-77.

16. Willson TM, Henke BR, Momtahen TM et al, 3-[4-(1,2-Diphenylbut-1-enyl)phenyl]acrylic acid: a nonsteroidal estrogen with functional selectivity for bone over uterus in rats. J Med Chem 1994;37:1550-2.

17. Fan M, Rickert EL, Chen L, et al. Characterization of molecular and structural determinants of selective estrogen receptor downregulators. Breast Cancer Res Treat 2007;103:37-44.

18. McDonnell DP, Wardell SE, Norris JD. Oral selective estrogen receptor downregulators (SERDs), a breakthrough endocrine therapy for breast cancer. J Med Chem 2015;58:4883-7.

19. Lai A, Kahraman M, Govek S, et al. Identification of GDC-0810 (ARN-810), an orally bioavailable selective estrogen receptor degrader (SERD) that demonstrates robust activity in tamoxifen-resistant breast cancer xenografts. J Med Chem 2015;58:4888-904

20. Joseph JD, Darimont B, Zhou W, et al. The selective estrogen receptor downregulator GDC-0810 is efficacious in diverse models of ER+ breast cancer. Elife 2016;5:e15828.

21. Weir HM, Bradbury RH, Lawson M, et al. AZD9496: an oral estrogen receptor inhibitor that blocks the growth of ER-positive and ESR1-mutant breast tumors in preclinical models. Cancer Res 2016;76:3307-18.

22. Toy W, Weir H, Razavi P, et al. Activating ESR1 mutations differentially affect the efficacy of ER antagonists. Cancer Discov 2017;7:277-87.

23. Robertson JF, Evans A, Henschen S, et al. A Randomized, Open-label, Presurgical, Window-of-Opportunity Study Comparing the Pharmacodynamic Effects of the Novel Oral SERD AZD9496 With Fulvestrant in Patients With Newly Diagnosed ER + HER2 - Primary Breast Cancer. Clin Cancer Res 2020. [Epub ahead of print].
Cite this article as: Wang G. Fulvestrant as a reference antiestrogen and estrogen receptor (ER) degrader in preclinical studies: treatment dosage, efficacy, and implications on development of new ER-targeting agents. Transl Cancer Res 2020;9(8):4464-4468. doi: 10.21037/tcr-20-2166 